Article

\title{
A Class of Nonlinear Fuzzy Variational Inequality Problems
}

\author{
Cunlin $\mathrm{Li}^{1}{ }^{1}$, Zhifu Jia ${ }^{2}\left(\mathbb{D}\right.$ and Yeong-Cheng Liou ${ }^{3,4, *}$
}

1 School of Management, Ningxia Key Laboratory of intelligent information and Big Data Processing, North Minzu University, Yinchuan 750021, China; bitlcl@163.com

2 School of Mathematics and Information Science, Ningxia Collaborative Innovation Center of Scientific Computation and Intelligent Information Processing, North Minzu University, Yinchuan 750021, China; jzflzbx@163.com

3 Department of Healthcare Administration and Medical Informatics, and Research Center of Nonlinear Analysis and Optimization, Kaohsiung Medical University, Kaohsiung 807, Taiwan

4 Department of Medical Research, Kaohsiung Medical University Hospital, Kaohsiung 807, Taiwan

* Correspondence: simplex_liou@hotmail.com

Received: 11 December 2018; Accepted: 3 January 2019; Published: 7 January 2019

\begin{abstract}
In this paper, we consider nonlinear variational inequality problems with fuzzy variables. The fuzzy variables were introduced to deal with the variational inequality containing noise for which historical data is not available. The fuzzy expected residual minimization (FERM) problems were established. We discussed the $S C^{1}$ property of the FERM model. Furthermore, results of convergence analysis were obtained based on an approximation model of the FERM model. The convergence of global optimal solutions and the convergence of stationary points were analysed.
\end{abstract}

Keywords: fuzzy variational inequalities; fuzzy set theory; expected residual functions; $S C^{1}$ property of FERM model; compact approximations

MSC: 49J53; 49J40; 65K10; 90C99

\section{Introduction}

In many real-world problems, examples contain some uncertain information, with examples including new stock, emergencies, military experiments, etc. For this uncertain information, often no historical data are available. In scientific fields, we also encountered similar uncertain situations, in which uncertainty contains noise that make measurements deviate from the correct, intended, or original values. Also, in these cases, experts cannot collect enough historical data to measure the noise. Zadeh [1] introduced fuzzy set theory, which provided efficacious ways to handle uncertain information. The uncertain information contained a belief degree, representing the strength with which we believe the event will happen. A fuzzy number is a kind of special fuzzy set and its operation was a key factor in processing fuzzy information. An interval-valued expectation of fuzzy numbers was given by Dubois and Prade [2,3] in 1987. The additive of the expectation was proved. They [4] also introduced possibility theory, about the introduction of qualitative and quantitative aspects, in 1998. In this paper, we define the possibility expectation values, possibility distribution function, and establish equivalence of fuzzy membership functions, which are consistent with the extension principle, and with the well-known definitions of expectation and variance in probability theory. The theory discussed in this paper was motivated by the principles in [2-13]. The concept of variational inequalities was introduced by Chang and Zhu [14] for fuzzy mappings in 1989. Soon afterwards, a new method was proposed by Huang [15] on a class of variational inequalities with fuzzy mappings. Huang [16] surveyed random generalized variational inclusions for random fuzzy mappings. In [17], the authors considered 
variational inequalities for fuzzy mappings. Moreover, an algorithm for fuzzy linear programming with piecewise linear membership functions was presented by Inuiguchi et al. [18]. A group of inequalities with fuzzy numbers, their membership functions are linear, were investigated by $\mathrm{Hu}$ and Fang [19]. They turned fuzzy inequalities into convex programming problems. Fang and $\mathrm{Hu}$ [20], and $\mathrm{Hu}$ [21] introduced and studied some fuzzy variational inequalities in a fuzzy environment, and proved the existence of the optimal solution for fuzzy variational inequalities by using the tolerance approach and the entropic regularization technique. In particular, in [22], Hu showed that solving the fuzzy variational inequalities are equivalent to solving a fuzzy generalized complementarity problem. Wang and Liao $[23,24]$ established fuzzy variational inequalities based on fuzzy convex cone and fuzzy resolution. For some related works associated with variational inequalities, discontinuous implicit quasi-variational inequalities with applications to fuzzy mappings, linear programming with fuzzy coefficients in constraints, maximum feasibility problems for continuous linear inequalities with applications to fuzzy linear programming, and bilevel decision with generalized semi-infinite optimization for fuzzy mappings as lower level problems, we refer the reader to [15,16,19-34]. In [35], the authors discussed the existence of a solution to the fuzzy variational-like inequality and some extended properties of the fuzzy variational-like inequality were found. Recently, Tang, Zhao, Wan and He [36] introduced two perturbation methods for a fuzzy mapping: in one method it is perturbed by a nonlinear mapping, and in the other it is perturbed by a vector. Under suitable coercivity conditions, they proved that the perturbed variational inequality with a fuzzy mapping has a solution. In this paper, we consider the variational inequality problem by using the expected value to solve fuzzy variables under possibility spaces. To begin with, let us recall that the fuzzy variational inequality problem is to find $\bar{x} \in S \subset R^{n}$ satisfying

$$
(x-\bar{x})^{T} F(\bar{x}, \xi) \geq 0 \forall x \in S, \text { a.s. }
$$

Here, $F: R \rightarrow R^{n}$ is a mapping, $\xi$ is fuzzy variable defined on possibility space, and a.s. is "almost surely" in possibility measure.

The fuzzy variational inequality problem is as follows:

$$
\mathscr{P}\left\{\gamma \in \Gamma \mid(x-\bar{x})^{T} F(\bar{x}, \xi(\gamma)) \geq 0, \forall x \in S\right\}=1, \bar{x} \in S,
$$

where $\xi(\gamma)$ is the fuzzy variable, $\Gamma$ is a nonempty set and $F: R^{n} \times \Gamma \rightarrow R^{n}$ is a mapping. The FERM method of the fuzzy variational inequality problem is to minimize an expected residual given by the regularized gap function

$$
g(x, \xi):=\max _{y \in S}\left\{(x-y)^{T} F(x, \xi)-\frac{\alpha}{2}\|x-y\|_{G}^{2}\right\},
$$

where $G_{n \times n}$ is symmetric and positive-definite, $\alpha$ is a positive, and $\|\cdot\|_{G}$ is $\|x\|_{G}=\sqrt{x^{T} G x}$ for $x \in R^{n}$. So the FERM model is

$$
\begin{aligned}
& \min \theta(x), \\
& \theta(x):=E[g(x, \xi)]=\int_{T} g(x, t) d \mathscr{P}(t), \\
& \text { s.t. } x \in S .
\end{aligned}
$$

Here, $E$ is the expectation of the fuzzy variable $\xi$ and $\mathscr{P}(t)$ stands for the fuzzy distribution function with respect to the fuzzy variable $\xi$.

The remainder of this article is arranged as follows. Basic information about fuzzy set theory will be reviewed in Section 2. In Section 3, we introduce the $S C^{1}$ property of function $\theta(x)$. In Section 4, we discuss the FERM model based on uncertainty theory in the case that the fuzzy event space is compact. Finally, we give conclusions in Section 5. 


\section{Preliminaries}

\subsection{Fuzzy Set Theory}

In this section, several basic results about fuzzy set theory are presented.

Definition 1. let $\Theta$ be a nonempty set and $\mathscr{F}$ the power set of $\Theta$. If the set function Pos $: \mathscr{F} \rightarrow[0,1]$ satisfies

(1) normality, $\mathscr{P}$ os $(\Theta)=1$,

(2) monotonicity, $\mathscr{P} o s(A) \leq \mathscr{P} o s(B), A \subset B, a, b \in \mathscr{F}$,

(3) for $i=1,2 \ldots, A_{i} \in \mathscr{F}$, we have $\mathscr{P}$ os $\left(\cup_{i} A_{i}\right)=\sup \mathscr{P}_{\text {os }}\left(A_{i}\right)$.

The element in $\mathscr{F}$ is called an event; the mapping is called a possibility measure; and $(\Theta, \mathscr{F}(\Theta), \mathscr{P}$ os $)$ is called a possibility measure space.

Theorem 1. The possibility measure is sub-additive. We have $\mathscr{P}_{\mathrm{os}}(A \cup B) \leq \mathscr{P}_{\mathrm{OS}}(A)+\mathscr{P}_{\mathrm{OS}}(B)$.

Proof. By Definition 1, we get $\mathscr{P}_{O S}(A \cup B)=\mathscr{P}_{O S}(A) \vee \mathscr{P}_{O S}(B) \leq \mathscr{P}_{O S}(A)+\mathscr{P}_{O S}(B)$.

Definition 2. Suppose $\xi$ is a fuzzy variable defined in the possibility space. $\xi$ is said to have a membership function $\mu_{\xi}(t)$, which is defined as follows:

$$
u_{\xi}(t)=\mathscr{P} \text { os }\{\theta \in \Theta \mid \xi(\theta)=t\}, t \in \mathscr{R},
$$

(denoted as $\mu(t)$ for convenience) with the premise that ambiguity does not occur.

Definition 3. $\xi$ is a fuzzy variable, its fuzzy distribution $\mathscr{P}(t)$ is defined by

$$
\mathscr{P}(t)=\mathscr{P} \text { os }\{\xi \leq t\}
$$

where $t$ is any real number.

Theorem 2. Suppose $\xi$ is a fuzzy variable defined in the possibility space. $\mu(t)$ is a membership function of $\xi$. For a real set $B$, we have

$$
\mathscr{P} o s(\xi \in B)=\sup \mu_{\xi}(t)
$$

Proof. From the definition of the axioms of the possibility measure, we have $\mathscr{P}$ os $(\xi \in B)=$ $\sup _{\xi \in B} \mathscr{P}_{O S}(\xi)=\sup _{\xi \in B} \mathscr{P} O S(\xi=t)=\sup \mu_{\xi}(t)$.

Remark 1. The fuzzy axiom system that we give and the fuzzy sets theory that Zadeh has put forward are consistent. In order to deal with fuzzy variables reasonably, we propose a fuzzy distribution function, which is consistent with membership function that Zadeh has given.

Let $\xi$ be a fuzzy variable defined in the possibility space and $\mu(t)$ be a membership function of $\xi$. The following equations are established

(1) $\mathscr{P} O s\{\xi \leq t\}=\sup _{y \leq t} \mu(y), t \in \mathscr{R}$,

(2) $\mathscr{P}$ os $\{\xi \geq t\}=\sup _{y \geq t} \mu(y), t \in \mathscr{R}$.

Next, we propose the concept of expected value. 
Definition 4. $\xi$ is a fuzzy variable defined in possibility space. If one of the following two integrals is finite, the expectation of $\xi$ is given by

$$
E[\tilde{\zeta}]=\int_{0}^{+\infty}\left\{1-\mathscr{P}_{O S}\{\xi \leq t\}\right\} d t-\int_{-\infty}^{0} \mathscr{P} \text { os }\{\xi \leq t\} d t
$$

Theorem 3. Let $\xi$ be a fuzzy variable with possibility distribution in $\mathscr{P}$. If its expectation exists, then

$$
E[\xi]=\int_{-\infty}^{+\infty} t d \mathscr{P}(t)
$$

where $\mathscr{P}(t)$ means $\mathscr{P}$ os $\{\xi \leq t\}$.

Proof. In fact, we can obtain

$$
\begin{aligned}
E[\xi] & =\int_{0}^{+\infty}\left\{1-\mathscr{P}_{O S}\{\xi \leq t\}\right\} d t-\int_{-\infty}^{0} \mathscr{P} O S\{\xi \leq t\} d t \\
& =\int_{0}^{+\infty}\{1-\mathscr{P}(t)\} d t-\int_{-\infty}^{0} \mathscr{P}(t) d t \\
& =\left.t(1-\mathscr{P}(t))\right|_{0} ^{+\infty}-\int_{0}^{+\infty} t d(1-\mathscr{P}(t))-\left.t \mathscr{P}(t)\right|_{-\infty} ^{0}+\int_{-\infty}^{0} t d \mathscr{P}(t) \\
& =\int_{0}^{+\infty} t d \mathscr{P}(t)+\int_{-\infty}^{0} t d \mathscr{P}(t) \\
& =\int_{-\infty}^{+\infty} t d \mathscr{P}(t) .
\end{aligned}
$$

Therefore the conclusion is reached.

Corollary 1. Let $\xi$ be an fuzzy variable and $\mathscr{P}(t)$ is its possibility distribution. If $f(t)$ is strictly monotone, then we have

$$
E[f(\xi)]=\int_{-\infty}^{+\infty} f(t) d \mathscr{P}(t)
$$

Proof. Substituting $\mathscr{P}(t)$ with $\alpha$, by Theorem 3 we have

$$
E[\xi]=\int_{-\infty}^{+\infty} t d \mathscr{P}(t)=\int_{0}^{1} \mathscr{P}^{-1}(\alpha) d \alpha .
$$

Let $\omega=f(\xi)$ with possibility distribution $P(t)$. Then we have

$$
E[\omega]=\int_{-\infty}^{+\infty} t d \mathscr{P}(t)=\int_{0}^{1} \mathscr{P}^{-1}(\alpha) d \alpha .
$$

Thus,

$$
E[f(\xi)]=\int_{0}^{1} \mathscr{P}^{-1}(\alpha) d \alpha=\int_{0}^{1} f\left(\mathscr{P}^{-1}(\alpha)\right) d \alpha=\int_{-\infty}^{+\infty} f(t) d \mathscr{P}(t) .
$$

The corollary is proved.

\subsection{Approximation Method}

Let a fuzzy event space $T$ be a nonempty compact set. From the continuity of $\left(F, \nabla_{x} F\right)$ and Theorem 4 of [13], we easily see that the function $\theta$ is continuously differentiable in $S$, and its derivative is as follows:

$$
\nabla \theta(x)=E\left[\nabla_{x} g(x, \xi)\right], \forall x \in S
$$


Since a fuzzy variable usually has no density function, $\mathscr{P}(x)$ is not usually differentiable in fuzzy theory. However, we can use the differentiable properties of $g(x, t)$. From [37], $g(\cdot, t)$ is continuously differentiable over $S$, satisfying

$$
\nabla_{x} g(x, t)=F(x, t)-\left(\nabla_{x} F(x, t)-\alpha G\right)(H(x, t)-x), \forall t \in T
$$

Definition 5 ([38]). Let $g(t)$ and $\mathscr{P}$ be real-valued bounded functions on a closed interval $[a, b]$. Taking a partition of the interval $a=x_{0}<x_{1}<x_{2}, \ldots<x_{n-1}<x_{n}=b$, and setting $\delta=\max _{1 \leq i \leq n}\left(t_{i}-t_{i-1}\right)$, we consider the Riemann sum $\lim _{\substack{\delta \rightarrow 0 \\ n \rightarrow \infty}} \sum_{i=1}^{n} g\left(z_{i}\right)\left[\mathscr{P}\left(t_{i}\right)-\mathscr{P}\left(t_{i-1}\right)\right]$, denoted by $\lim _{\substack{\delta \rightarrow 0 \\ n \rightarrow \infty}} \sum_{i=1}^{n} g\left(z_{i}\right) \Delta \mathscr{P}\left(t_{i}\right)$ (where $\left.t_{i-1} \leq z_{i} \leq t_{i}, i=1,2, \cdots, n\right)$. If the sum tends to a fixed number I as $\delta \rightarrow 0$, then I is called the Stieltjes integral, or sometimes the Riemann-Stieltjes integral. The Stieltjes integral of $g$ with respect to $t$ is denoted $\int_{a}^{b} g(t) d \mathscr{P}(t)$ or sometimes simply by $\int g d \mathscr{P}$.

Lemma 1 ([38]). If the function $g(t)$ is continuous on $[a, b]$ and $\mathscr{P}(t)$ is monotonic nonincreasing or nondecreasing function, then $\int_{0}^{1} g(t) d \mathscr{P}(t)$ exists.

Let $\lambda_{\max }$ and $\lambda_{\min }$ represent the largest and smallest eigenvalues of the positive definite matrix $G$, respectively. For any $x \in R^{n}$, we have

$$
\sqrt{\lambda_{\min }}\|x\| \leq\|x\|_{G} \leq \sqrt{\lambda_{\max }}\|x\|
$$

Let $\|A\|$ denote the spectral norm and $\|A\|_{\mathscr{F}}$ denote the Frobenius matrix norm of matrix $A$. We know that

$$
\|A\| \leq\|A\|_{\mathscr{F}},
$$

and

$$
\|A\|_{\mathscr{F}} \leq \sum_{j=1}^{n}\left\|A_{j}\right\|,
$$

where $A_{j}$ is the jth column vector of $A$.

For convenience, we give the relationship between $\|A\|$ and $\|A\|_{\mathscr{F}}$ and the relationship between $\|x\|$ and $\|x\|_{G}$ in advance. These definitions and properties will be used in the later theorems.

\section{The $S C^{1}$ Property of $\theta(x)$}

Definition 6 ([39]). A function $g: R^{n} \rightarrow R$ is called an $S C^{1}$ function if its gradient is semi-smooth and $g$ is continuously differentiable.

Lemma 2 ([39]). Let $x_{0} \in S$. Suppose that

(1) there exists an integrable function $\kappa: R \rightarrow R_{+}$and a neighborhood $N\left(x_{0}\right)$ of $x_{0}$ satisfying

$$
\left|g\left(x_{0}, t\right)-g(x, t)\right| \leq k(t)\left\|x_{0}-x\right\|, \forall x \in N\left(x_{0}\right) \text {, a.e. } t \in T ;
$$

(2) $g(x, t)$ is semi-smooth at $x_{0}$ for a.e. $t \in T$;

(3) there exist an integrable function $\zeta: R \rightarrow R_{+}$and a neighborhood $N(0)$ of $0 \in R^{n}$ satisfying

$$
\frac{\left\|g^{\prime}\left(x_{0}+h, t\right)-g^{\prime}\left(x_{0}, t\right)\right\|}{\|h\|} \leq \zeta(t), \forall h \in N(0) \text {, a.e. } t \in T .
$$

Then $\theta(x)$ is semi-smooth at $x_{0}$. 
Theorem 4. Let $x_{0} \in S, N\left(x_{0}\right)$ be a neighborhood of $x_{0}$. Suppose that

(1) there exist an integrable function $\kappa: R \rightarrow R_{+}$, such that $\int_{T} \kappa(t) d \mathscr{P}(t)<+\infty$, satisfying

$$
\left|g\left(x_{1}, t\right)-g\left(x_{2}, t\right)\right| \leq \kappa(t)\left\|x_{1}-x_{2}\right\|, \forall x_{1}, x_{2} \in N\left(x_{0}\right) \text {, a.e. } t \in T .
$$

(2) For a.e. $t \in T, g(x, t)$ is $S C^{1}$ function on $N\left(x_{0}\right)$. There is nonnegative function such that $\int_{T} L(t) d \mathscr{P}(t)<$ $+\infty$, satisfying $\nabla g(x, t)$ is Lipschitz continuous,

$$
\left|\nabla g\left(x_{1}, t\right)-\nabla g\left(x_{2}, t\right)\right| \leq L(t)\left\|x_{1}-x_{2}\right\|
$$

(3) There exists an integrable function $\zeta: R \rightarrow R_{+}$and $N(0)$ of $0 \in R^{n}$ satisfying

$$
\frac{\left\|s^{\prime}\left(x_{0}+h, t\right)-s^{\prime}\left(x_{0}, t\right)\right\|}{\|h\|} \leq \zeta(t), \forall h \in N(0), \text { a.e. } t \in T,
$$

where $s(x, t)=\nabla g(x, t)$, and $\nabla$ represents gradient.

Then $\theta(x)$ is $S C^{1}$ function on $N\left(x_{0}\right)$.

Proof. By condition (2), the gradient of $g(\cdot, t)$ is semi-smooth, and $g(\cdot, t)$ is continuous and differentiable. It is easy to see that $s(x, t)$ is directional differentiable, Lipschitz continuous, and

$$
s^{\prime}\left(x_{0}+h, t\right)-s^{\prime}\left(x_{0}, t\right)=o(\|h\|)^{\prime} .
$$

Next, we show $\theta(x)$ is $S C^{1}$ function. First, we show $\theta(x)$ is differentiable at $x_{0}$. By definition of $\theta(x)$ and condition (1), we obtain

$$
\begin{aligned}
\left|\theta\left(x_{1}\right)-\theta\left(x_{2}\right)\right| & =\left|\int_{T} g\left(x_{1}, t\right) d \mathscr{P}(t)-\int_{T} g\left(x_{2}, t\right) d \mathscr{P}(t)\right| \\
& \leq \int_{T}\left|g\left(x_{1}, t\right)-g\left(x_{2}, t\right)\right| d \mathscr{P}(t) \\
& \leq\left\|x_{1}-x_{2}\right\| \int_{T} \kappa(t) d \mathscr{P}(t)
\end{aligned}
$$

for $\forall x_{1}, x_{2} \in N\left(x_{0}\right)$ and $t \in T$. So $\theta(x)$ is differentiable at $x_{0}$. By condition (1), for $h \in R^{n}$

$$
l^{-1}\left|g\left(x_{0}+l h, t\right)-g\left(x_{0}, t\right)\right| \leq \kappa(t)\|h\| .
$$

Note that

$$
\begin{aligned}
\lim _{l \rightarrow 0} \frac{\theta\left(x_{0}+l h, t\right)-\theta\left(x_{0}, t\right)}{l} & =\lim _{l \rightarrow 0} \frac{\int_{T} g\left(x_{0}+l h, t\right) d \mathscr{P}(t)-\int_{T} g\left(x_{0}, t\right) d \mathscr{P}(t)}{l} \\
& =\int_{T} \lim _{l \rightarrow 0} \frac{g\left(x_{0}+l h, t\right)-g\left(x_{0}, t\right)}{l} d \mathscr{P}(t),
\end{aligned}
$$

so $\nabla \theta(x)$ is directional differentiable, denoted

$$
\theta^{\prime}\left(x_{0} ; h\right)=\int_{T} g^{\prime}\left(x_{0} ; h, t\right) d \mathscr{P}(t)
$$

Since $g^{\prime}\left(x_{0} ; h, t\right)=\nabla g^{\prime}\left(x_{0}, t\right)^{H} h, \theta^{\prime}\left(x_{0} ; h\right)$ is linear with respect to $h$, from Lipschitz continuity and Gateaux differentiablity of $\theta(x), \theta(x)$ is Frechet differentiable.

Note that $\theta(x)$ is differentiable at $x_{0}, \nabla \theta\left(x_{0}\right)=\int_{T} \nabla g\left(x_{0}, t\right) d \mathscr{P}(t)$, and $\nabla g(x, t)$ is semi-smooth at $x_{0}$ and is Lipschitz continuous. We get 


$$
\begin{aligned}
\left|\nabla \theta\left(x_{1}\right)-\nabla \theta\left(x_{2}\right)\right| & =\left|\int_{T} \nabla g\left(x_{1}, t\right) d \mathscr{P}(t)-\int_{T} \nabla g\left(x_{2}, t\right) d \mathscr{P}(t)\right| \\
& \leq \int_{T}\left|\nabla g\left(x_{1}, t\right)-\nabla g\left(x_{2}, t\right)\right| d \mathscr{P}(t) \\
& \leq\left\|x_{1}-x_{2}\right\| \int_{T} L(t) d \mathscr{P}(t),
\end{aligned}
$$

where $\int_{T} L(t) d \mathscr{P}(t)<+\infty$, so $\forall x_{1}, x_{2} \in N\left(x_{0}\right)$, a.e. $t \in T, \nabla \theta(x)$ is Lipschitz continuous.

Finally, we will show $\nabla \theta(x)$ is semi-smooth. Let $v(x):=\nabla \theta(x)=\int_{T} \nabla g(t) d \mathscr{P}(t)$, we need to show $\nabla \theta(x)$ is directional differentiable and $v^{\prime}(x+h)-v^{\prime}(x)=o(\|h\|)$.

By condition (3), we have

$$
\begin{aligned}
\frac{\left\|v^{\prime}(x+h)-v^{\prime}(x)\right\|}{\|h\|} & =\int_{T} \frac{\left\|\nabla g^{\prime}(x+h, t)-\nabla g^{\prime}(x, t)\right\|}{\|h\|} d \mathscr{P}(t) \\
& =\int_{T} \frac{\left\|s^{\prime}(x+h, t)-s^{\prime}(x, t)\right\|}{\|h\|} d \mathscr{P}(t) \\
& \leq \int_{T} \zeta(t) d \mathscr{P}(t) \\
& <+\infty .
\end{aligned}
$$

Since $s^{\prime}(x, t)$ is semi-smooth, $\lim _{h \rightarrow 0} \frac{\left\|v^{\prime}(x+h)-v^{\prime}(x)\right\|}{\|h\|}=\int_{T} \lim _{h \rightarrow 0} \frac{\left\|v^{\prime}(x+h)-v^{\prime}(x)\right\|}{\|h\|} d \mathscr{P}(t)=0$. By Lemma 2, $\nabla \theta(x)$ is semi-smooth. So $\theta(x)$ is $S C^{1}$ on $N\left(x_{0}\right)$.

\section{Convergence of the FERM Model}

Definition 7. Define the minimum of $\theta^{n}(x)$ as follows:

$$
\min \theta^{n}(x)=\sum_{t^{i} \in T_{\delta}} g\left(x, z_{i}\right) \Delta \mathscr{P}\left(t_{i}\right)
$$

where $T_{\delta}=\left\{t_{i} \mid i=1,2, \cdots, n_{\delta}\right\}$ is a set satisfying $n_{\delta} \rightarrow \infty$ as $\delta \rightarrow 0$.

We study the behavior of the approximations to the FERM problem (3) as follow

$$
\begin{aligned}
& \min \theta^{n}(x)=\sum_{t_{i} \in T_{\delta}} g\left(x, z_{i}\right) \Delta \mathscr{P}\left(t_{i}\right), \\
& \text { s.t. } x \in S .
\end{aligned}
$$

We consider the limiting behavior of problems (10) below.

\subsection{Convergence of Global Optimal Solutions}

Let $S^{*}$ and $S_{n}^{*}$ be the sets of optimal solutions of problems (3) and (10).

Lemma 3. $\theta(x)=\lim _{\substack{\delta \rightarrow 0 \\ n \rightarrow \infty}} \theta^{n}(x)$ holds for any given $x \in S$.

Proof. Since

$$
\theta(x)=E[g(x, \xi)]=\int_{T}(x, t) d \mathscr{P}(t)
$$

while

$$
\theta^{n}(x)=\sum_{t^{i} \in T_{\delta}} g\left(x, z_{i}\right) \Delta \mathscr{P}\left(t_{i}\right)
$$


therefore $\lim _{\substack{\delta \rightarrow 0 \\ n \rightarrow \infty}} \sum_{t_{i} \in T_{\delta}} g\left(x, z_{i}\right) \Delta \mathscr{P}\left(t_{i}\right)=\int_{T} g(x, t) d \mathscr{P}(t)$, so $\theta(x)=\lim _{\substack{\delta \rightarrow 0 \\ n \rightarrow \infty}} \theta^{n}(x)$.

Theorem 5. Let $\bar{x}$ be an accumulation point of $\left\{x^{n}\right\}, x^{n} \in S_{n}^{*}$, then $\bar{x} \in S^{*}$.

Proof. Let $\lim _{n \rightarrow \infty} x_{n}=\bar{x}$. It holds that $\bar{x} \in S$. Let $B \subseteq S$ be a compact convex set, and $x^{n} \in B$. By the continuity of $\nabla_{x} g$ on the compact set $B \times T, \exists C \in R^{+}$and we have

$$
\left\|\nabla_{x} g(x, z)\right\| \leq C, \forall(x, z) \in B \times T .
$$

For each $x^{n}$ and each $z_{i}$, the next equation holds from the mean-value theorem:

$$
g\left(x^{n}, z_{i}\right)-g\left(\bar{x}, z_{i}\right)=g_{x}^{\prime}\left(y^{n i}, z_{i}\right)^{T}\left(x^{n}-\bar{x}\right),
$$

where $y^{n i}=\alpha_{n i} x^{n}+\left(1-\alpha_{n i}\right) \bar{x} \in B$ with $\alpha_{n i} \in[0,1]$. It then follows from Lemma 3 that

$$
\begin{aligned}
\left|\theta^{n}\left(x^{n}\right)-\theta^{n}(\bar{x})\right| & =\left|\sum_{t^{i} \in T_{\delta}}\left(g\left(x^{n}, z_{i}\right)-g\left(\bar{x}, z_{i}\right)\right) \Delta \mathscr{P}\left(t_{i}\right)\right| \\
& \leq \sum_{t_{i} \in T_{\delta}}\left|\left(g\left(x^{n}, z_{i}\right)-g\left(\bar{x}, z_{i}\right)\right)\right| \Delta \mathscr{P}\left(t_{i}\right) \\
& =\sum_{t_{i} \in T_{\delta}}\left|\nabla_{x} g\left(y^{n i}, z_{i}\right)^{T}\left(x^{n}-\bar{x}\right)\right| \Delta \mathscr{P}\left(t_{i}\right) \\
& \leq \sum_{t_{i} \in T_{\delta}}\left\|\nabla_{x} g\left(y^{n i}, z_{i}\right)\right\| \cdot\left\|\left(x^{n}-\bar{x}\right)\right\| \Delta \mathscr{P}\left(t_{i}\right) \\
& \leq C\left\|x^{n}-\bar{x}\right\| \sum_{t_{i} \in T_{\delta}} \Delta \mathscr{P}\left(t_{i}\right) \\
& \stackrel{n \rightarrow \infty}{\longrightarrow} 0,
\end{aligned}
$$

noting that

$$
\left|\theta^{n}\left(x^{n}\right)-\theta(\bar{x})\right| \leq\left|\theta^{n}\left(x^{n}\right)-\theta^{n}(\bar{x})\right|+\left|\theta^{n}(\bar{x})-\theta(\bar{x})\right| .
$$

From Lemma 3 and (12), it holds that

$$
\lim _{\substack{\delta \rightarrow 0 \\ n \rightarrow \infty}} \theta^{n}\left(x^{n}\right)=\theta(\bar{x})
$$

Since, for each sufficiently large $n, x^{n} \in S_{n}^{*}$, which means that there exists $\epsilon>0$ such that

$$
\theta^{n}\left(x^{n}\right) \leq \theta^{n}(x)+\epsilon .
$$

This holds for any $x \in S$. Letting $n \rightarrow \infty$ in (14) and taking into account (13) and Lemma 2, $\theta(\bar{x}) \leq \theta(x)+\epsilon$ holds implies $\bar{x} \in S^{*}$.

\subsection{Convergence of Stationary Points}

Theorem 6. Let $\lim _{n \rightarrow \infty} x^{n}=\bar{x}$. Then,

$$
\lim _{\substack{\delta \rightarrow 0 \\ n \rightarrow \infty}} \nabla \theta^{n}\left(x^{n}\right)=\nabla \theta(\bar{x})
$$

Proof. Let $B \subseteq S$ be a compact convex set and $x^{n} \in B$. Because $F_{x}^{\prime}, g_{x}^{\prime}, H$, and $\left(F_{j}\right)_{x}^{\prime \prime}$ are continuous on the compact set $B \times T$, for any $(x, z) \in B \times T, \exists C \geq \sup \left\{\left\|x^{n}\right\|, n=1,2, \ldots\right\}$ satisfying

$$
\left\|F_{x}^{\prime}(x, z)\right\| \leq C,
$$




$$
\begin{gathered}
\|H(x, z)\| \leq C, \\
\left\|\left(F_{j}\right)_{x}^{\prime \prime}(x, z)\right\| \leq C, j=1, \cdots, n,
\end{gathered}
$$

where $\left(F_{j}\right)_{x}^{\prime \prime}(x, z)$ denotes the Hessian matrix of $F_{j}(x, z)$ with respect to $x$.

We first show that

$$
\lim _{\substack{\delta \rightarrow 0 \\ n \rightarrow \infty}} \sum_{t^{i} \in T_{n}} \Delta \mathscr{P}\left(t^{i}\right)\left\|\nabla_{x} F\left(x^{n}, z_{i}\right)-\nabla_{x} F\left(\bar{x}, z_{i}\right)\right\|=0 .
$$

In fact, from (7) and (8), we have

$$
\begin{aligned}
\sum_{t_{i} \in T_{\delta}} \Delta \mathscr{P}\left(t_{i}\right)\left\|F_{x}^{\prime}\left(x^{n}, z_{i}\right)-F_{x}^{\prime}\left(\bar{x}, z_{i}\right)\right\| & \leq \sum_{t_{i} \in T_{\delta}} \Delta \mathscr{P}\left(t_{i}\right)\left\|F_{x}^{\prime}\left(x^{n}, z_{i}\right)-F_{x}^{\prime}\left(\bar{x}, z_{i}\right)\right\|_{F} \\
& \leq \sum_{j=1}^{n} \sum_{t_{i} \in T_{\delta}} \Delta \mathscr{P}\left(t_{i}\right)\left\|\left(F_{j}\right)_{x}^{\prime}\left(x^{n}, z_{i}\right)-\left(F_{j}\right)_{x}^{\prime}\left(\bar{x}, z_{i}\right)\right\|
\end{aligned}
$$

From the mean-value theorem, for any fixed $j$ and for each $x^{n}$ and $z^{i}$, there exists $y^{n i j}=\alpha_{n i j} x^{n}+$ $\left(1-\alpha_{n i j}\right) \bar{x} \in B$ with $\alpha_{n i j} \in[0,1]$ such that

$$
\begin{aligned}
\sum_{j=1}^{n} \sum_{t_{i} \in T_{\delta}} \Delta \mathscr{P}\left(t_{i}\right)\left\|\left(F_{j}\right)_{x}^{\prime}\left(x^{n}, z_{i}\right)-\left(F_{j}\right)_{x}^{\prime}\left(\bar{x}, z_{i}\right)\right\| & \leq \sum_{j=1}^{n} \sum_{t^{i} \in T_{\delta}} \Delta \mathscr{P}\left(t_{i}\right)\left\|\left(F_{j}\right)_{x}^{\prime \prime}\left(y^{k i j}, z_{i}\right)\right\|\left\|x^{n}-\bar{x}\right\| \\
& \leq C\left\|x^{n}-\bar{x}\right\| \sum_{t_{i} \in T_{\delta}} \Delta \mathscr{P}\left(t_{i}\right) \\
& \stackrel{n \rightarrow \infty}{\longrightarrow} 0,
\end{aligned}
$$

where the second inequality follows from (1). We have (18) immediately from (19) and (20). In a similar way, we can show that

$$
\lim _{\substack{\delta \rightarrow 0 \\ n \rightarrow \infty}} \sum_{t_{i} \in T_{\delta}} \Delta \mathscr{P}\left(t_{i}\right)\left\|F\left(x^{n}, z_{i}\right)-F\left(\bar{x}, z_{i}\right)\right\|=0 .
$$

From the nonexpansive property of $\operatorname{Proj}_{S, G}$, (6) and (21), it holds that

$$
\begin{aligned}
& \sum_{t_{i} \in T_{\delta}} \Delta \mathscr{P}\left(t_{i}\right)\left\|H\left(\bar{x}, z_{i}\right)-H\left(x^{n}, z_{i}\right)\right\| \\
& \leq \lambda_{\min }^{-\frac{1}{2}} \sum_{t^{i} \in T_{\delta}} \Delta \mathscr{P}\left(t_{i}\right)\left\|H\left(\bar{x}, z_{i}\right)-H\left(x^{n}, z_{i}\right)\right\|_{G} \\
& \leq \lambda_{\min }^{-\frac{1}{2}} \sum_{t^{i} \in T_{\delta}} \Delta \mathscr{P}\left(t_{i}\right) \|\left(\bar{x}-\alpha^{-1} G^{-1} F\left(\bar{x}, z_{i}\right)-\left(x^{n}-\alpha^{-1} G^{-1} F\left(x^{n}, z_{i}\right) \|_{G}\right.\right. \\
& \leq \lambda_{\max }^{\frac{1}{2}} \lambda_{\min }^{-\frac{1}{2}} \sum_{t^{i} \in T_{\delta}} \Delta \mathscr{P}\left(t_{i}\right)\left(\left\|x^{k}-\bar{x}\right\|+\alpha^{-1}\left\|G^{-1}\right\|\left\|F\left(x^{n}, z_{i}\right)-F\left(\bar{x}, z_{i}\right)\right\|\right) \\
& \stackrel{n \rightarrow \infty}{\longrightarrow} 0 .
\end{aligned}
$$


On the other hand, by (15), (16), (18) and (22), we have

$$
\begin{aligned}
& \sum_{t_{i} \in T_{\delta}} \Delta \mathscr{P}\left(t_{i}\right)\left\|F_{x}^{\prime}\left(x^{n}, z_{i}\right) H\left(x^{n}, z_{i}\right)-F_{x}^{\prime}\left(\bar{x}, z_{i}\right) H\left(\bar{x}, z_{i}\right)\right\| \\
& =\sum_{t^{i} \in T_{\delta}} \Delta \mathscr{P}\left(t^{i}\right) \| F_{x}^{\prime}\left(x^{n}, z_{i}\right)\left(H\left(x^{n}, z_{i}\right)-H\left(\bar{x}, z_{i}\right)\right) \\
& +\left(F_{x}^{\prime}\left(x^{n}, z_{i}\right)-F_{x}^{\prime}\left(\bar{x}, z_{i}\right)\right)\left\|H\left(\bar{x}, z_{i}\right)\right\| \\
& \leq \sum_{t_{i} \in T_{\delta}} \Delta \mathscr{P}\left(t_{i}\right)\left\|F_{x}^{\prime}\left(x^{n}, z_{i}\right)\right\| H\left(x^{n}, z_{i}\right)-H\left(\bar{x}, z_{i}\right) \| \\
& +\left\|F_{x}^{\prime}\left(x^{n}, z_{i}\right)-F_{x}^{\prime}\left(\bar{x}, z_{i}\right)\right\|\left\|H\left(\bar{x}, z_{i}\right)\right\| \\
& \leq C \cdot \sum_{t_{i} \in T_{\delta}} \Delta \mathscr{P}\left(t_{i}\right)\left(\|\left(H\left(x^{n}, z_{i}\right)-H\left(\bar{x}, z_{i}\right)\|+\| F_{x}^{\prime}\left(x^{n}, z_{i}\right)-F_{x}^{\prime}\left(\bar{x}, z_{i}\right) \|\right)\right. \\
& \stackrel{n \rightarrow \infty}{\longrightarrow} 0 .
\end{aligned}
$$

Noting that $C \geq \sup \left\{\left\|x^{n}\right\|, k=1,2, \ldots\right\}$, we have from (15) and (18) that

$$
\begin{aligned}
& \sum_{t_{i} \in T_{\delta}} \Delta \mathscr{P}\left(t_{i}\right)\left\|F_{x}^{\prime}\left(x^{n}, z_{i}\right) x^{n}-F_{x}^{\prime}\left(\bar{x}, z_{i}\right) \bar{x}\right\| \\
& \leq \sum_{t^{i} \in T_{\delta}} \Delta \mathscr{P}\left(t_{i}\right)\left(\left\|F_{x}^{\prime}\left(x^{n}, z_{i}\right) x^{n}-F_{x}^{\prime}\left(\bar{x}, z_{i}\right) \bar{x}\right\|\left\|x^{n}\right\|\right)+\left\|F_{x}^{\prime}\left(\bar{x}, z_{i}\right)\right\|\left\|x^{n}-\bar{x}\right\| \\
& \leq C \cdot \sum_{t^{i} \in T_{\delta}} \Delta \mathscr{P}\left(t_{i}\right)\left(\left\|F_{x}^{\prime}\left(x^{n}, z_{i}\right)-F_{x}^{\prime}\left(\bar{x}, z_{i}\right)\right\|+\left\|x^{n}-\bar{x}\right\|\right) \\
& \stackrel{n \rightarrow \infty}{\longrightarrow} 0 .
\end{aligned}
$$

Thus, it follows from (5), (21)-(24) that

$$
\begin{aligned}
\left\|\left(\theta_{x}^{\prime}\right)^{n}\left(x^{n}\right)-\left(\theta_{x}^{\prime}\right)^{n}(\bar{x})\right\| & \leq \sum_{t_{i} \in T_{\delta}} \Delta \mathscr{P}\left(t_{i}\right)\left(\left\|F\left(x^{n}, z_{i}\right)-F\left(\bar{x}, z_{i}\right)\right\|\right. \\
& +\left\|F_{x}^{\prime}\left(x^{n}, z_{i}\right) H\left(x^{n}, z_{i}\right)-F_{x}^{\prime}\left(\bar{x}, z_{i}\right) H\left(\bar{x}, z_{i}\right)\right\| \\
& +\left\|F_{x}^{\prime}\left(x^{n}, z_{i}\right) x^{n}-F_{x}^{\prime}\left(\bar{x}, z_{i}\right) \bar{x}\right\| \\
& \left.+\alpha\|G\|\left\|H\left(\bar{x}, z_{i}\right)-H\left(x^{n}, z_{i}\right)\right\|+\alpha\|G\|\left\|x^{n}-\bar{x}\right\|\right) \\
& \stackrel{n \rightarrow \infty}{\longrightarrow} 0 .
\end{aligned}
$$

In a similar way with Lemma 3, we have $\lim _{\substack{\delta \rightarrow 0 \\ n \rightarrow \infty}}\left(\theta_{x}^{\prime}\right)^{n}(\bar{x})=\theta_{x}^{\prime}(\bar{x})$, so we get the conclusion of this lemma immediately from (25).

Now, we consider the limiting behavior of the stationary points of problems (10).

Definition 8. Assume that $S=\left\{x \in R^{n} \mid \tau_{i}(x) \leq 0, \tau_{i}: R^{n} \rightarrow R, i=1,2, \cdots, m\right\}$, where, $\tau_{i}(x)$ are all continuous and differentiable convex functions. If a Lagrange multiplier vector $\mu^{n} \in R^{m}$ satisfies

$$
\begin{gathered}
\left(\theta_{x}^{\prime}\right)^{n}\left(x^{n}\right)+\sum_{i=1}^{m} \mu_{i}^{n}\left(\left(\tau_{i}\right)_{x}^{\prime}\right)\left(x^{n}\right)=0, \\
0 \leq \mu^{n}, \tau\left(x^{n}\right) \leq 0 \text { and }\left(\mu^{n}\right)^{T} \tau\left(x^{n}\right)=0,
\end{gathered}
$$

then the point $x^{n}$ is a stationary point of (10).

If there is a Lagrange multiplier vector $\bar{\mu} \in R^{m}$ satisfing

$$
\theta_{x}^{\prime}(\bar{x})+\sum_{i=1}^{m} \bar{\mu}_{i}\left(\left(\tau_{i}\right)_{x}^{\prime}\right)(\bar{x})=0
$$




$$
0 \leq \bar{\mu}, \tau(\bar{x}) \leq 0 \text { and }(\bar{\mu})^{T} \tau(\bar{x})=0,
$$

then $\bar{x}$ is a stationary point of problem (3).

Definition 9. If $\exists y \in R^{n}$ such that $\tau_{i}(y)<0, i=1,2, \cdots, m$, then the Slater's constraint qualification holds.

Theorem 7. Let $x^{n}$ be stationary point of (10) and $\bar{x}$ be an accumulation point of $\left\{x^{n}\right\}$, then $\bar{x}$ is stationary point of (3) under the Slater's constraint qualification condition.

Proof. Let $\lim _{n \rightarrow \infty} x^{n}=\bar{x}$ and $\mu^{n}$ be corresponding multiplier vectors of (26) and (27).

(i) Firstly, we demonstrate that $\left\{\mu^{n}\right\}$ is bounded. For convenience, denote

$$
v_{n}:=\sum_{i=1}^{m} \mu_{i}^{n}
$$

If $\left\{\mu^{n}\right\}$ is unbounded, then $\lim _{k \rightarrow \infty} v_{n}=+\infty$. Further, taking a subsequence of $\mu^{n}$ such that $\bar{\mu}_{i}:=\lim _{n \rightarrow \infty} \frac{\mu_{i}^{n}}{v_{n}}(i=1,2, \cdots, m)$ exist. For every $i \notin \mathrm{Y}(\bar{x}):=\left\{i \mid \tau_{i}(\bar{x})=0,1 \leq i \leq m\right\}$, we have $\tau_{i}(\bar{x}) \leq 0$ by (27), while $\left(\bar{\mu}_{i}\right)^{T} \tau_{i}(\bar{x})=0$, so it is easy to obtain that $\mu_{i}^{*}=0$. We then have from (30) that

$$
\sum_{i \in Y(\bar{x})} \bar{\mu}_{i}=\sum_{i=1}^{m} \bar{\mu}_{i}=1
$$

Note that $\left(\left(\tau_{i}\right)_{x}^{\prime}\right)$ is continuous for each $i$ and, by Theorem $5,\left\{\left(\theta_{x}^{\prime}\right)^{n}\left(x^{n}\right)\right\}$ is convergent. Then, dividing (26) by $v_{n}$ and taking a limit, because of $\lim _{n \rightarrow \infty} v_{n}=+\infty$, so $\lim _{n \rightarrow \infty} \frac{\left(\theta_{x}^{\prime}\right)^{n}\left(x^{n}\right)}{v_{n}} \rightarrow 0$ and we obtain

$$
\sum_{i \in Y(\bar{x})} \bar{\mu}_{i}\left(\left(\tau_{i}\right)_{x}^{\prime}\right)(\bar{x})=\sum_{i=1}^{m} \bar{\mu}_{i}\left(\left(\tau_{i}\right)_{x}^{\prime}\right)(\bar{x})=0
$$

From the Slater's constraint qualification, $\exists y \in R^{n}$ satisfy $\tau_{i}(y)<0$. Moreover, $\tau_{i}, i=1,2, \cdots, m$ is convex, It holds that

$$
(y-\bar{x})^{T}\left(\left(\tau_{i}\right)_{x}^{\prime}\right)(\bar{x}) \leq \tau_{i}(y)-\tau_{i}(\bar{x})=\tau_{i}(y)<0, \forall i \in \mathrm{Y}(\bar{x})
$$

Since $\bar{\mu}_{i} \geq 0$ for each $i$ by (27), following from (32) we get $\bar{\mu}_{i}\left(\left(\tau_{i}\right)_{x}^{\prime}\right)(\bar{x})=0$, from (33) we get $\left(\left(\tau_{i}\right)_{x}^{\prime}\right)(\bar{x}) \neq 0$, so we naturally get that $\bar{\mu}_{i}=0, i \in \mathrm{Y}(\bar{x})$. This contradicts (31), so $\left\{\mu^{n}\right\}$ is bounded.

(ii) By (i), in $\mu^{n}$ exists a subsequence, we still denote it by $\mu^{n}$ such that $\bar{\mu}:=\lim _{n \rightarrow \infty} \mu^{n}$ because $\tau_{i}$ and $\left(\left(\tau_{i}\right)_{x}^{\prime}\right)$ are all continuous for each $i$. From Theorem 5 , we have

$$
\lim _{n \rightarrow \infty}\left(\theta_{x}^{\prime}\right)^{n}(\bar{x})=\theta_{x}^{\prime}(\bar{x})
$$

Then (28) and (29) hold by taking a limit on both sides of (26) and (27). Thus, $\bar{x}$ is a stationary point of problem (3).

\section{Conclusions}

We investigated the nonlinear variational inequality with fuzzy variable. The fuzzy nonlinear variational inequality model was established. $S C^{1}$ property of the FERM model The convergence of the algorithm of the fuzzy model was presented, based on the fuzzy event space being compact. The convergence of global optimal solutions and the convergence of stationary points were analysed. In the future, we will study the $D$ gap function and its applications, fuzzy quasi-variational inequalities, and fuzzy weighted variational inequalities. 
Author Contributions: All the authors have contributed equally to this paper. All the authors have read and approved the final manuscript.

Funding: Cunlin Li was supported by the National Natural Science Foundation of China (No. 71561001) and the Key Scientific Research Projects in 2017 at North Minzu University (2017KJ13). Zhifu Jia was supported by First-Class Disciplines Foundation of Ningxia (Grant No.NXYLXK2017B09) and Governance and social management research center of Northwest Ethnic regions-one of the Key Social Science Research Base of State Ethnic Affairs Commission of China. Yeong-Cheng Liou was supported in part by the grand form Kaohsiung Medical University Research Foundation (KMU-M108002), Taiwan-Thailand joint grant MOST 106-2218-E-040-001-MY2 and Taiwan-Russian joint grant MOST 106-2923-E-039-001-MY3.

Conflicts of Interest: The authors declare no conflict of interest.

\section{References}

1. Zadeh, L.-A. Fuzzy sets. Inform. Control 1965, 8, 338-353. [CrossRef]

2. Dubois, D.; Prade, H. The mean value of a fuzzy number. Fuzzy Sets Syst. 1987, 24, 279-300. [CrossRef]

3. Dubois, D.; Prade, H. Fussy Sets and Systems; Academic Press: Cambridge, MA, USA, 1980.

4. Dubois, D.; Prade, H. Possibility Theory: Qualitative and Quantitative Aspects. Quantified Representation of Uncertainty and Imprecision; Springer: Dordrecht, The Netherlands, 1998; pp. 169-226.

5. Cho, S.-Y.; Qin, X.; Yao, J.-C.; Yao, Y. Viscosity approximation splitting methods for monotone and nonexpansive operators in Hilbert spaces. J. Nonlinear Convex Anal. 2018, 19, 251-264.

6. Dubois, D.; Prade, H. Comment on tolerance analysis using fuzzy sets and a procedure for multiple aspect decision making. Internat. J. Syst. Sci. 1978, 9, 357-360. [CrossRef]

7. Yao, Y.; Chen, R.; Xu, H.-K. Schemes for finding minimum-norm solutions of variational inequalities. Nonlinear Anal. 2010, 72, 3447-3456. [CrossRef]

8. Yao, Y.; Liou, Y.-C.; Kang, S.-M. Approach to common elements of variational inequality problems and fixed point problems via a relaxed extragradient method. Comput. Math. Appl. 2010, 59, 3472-3480. [CrossRef]

9. Dubois, D.; Prade, H. Additions of interactive fuzzy numbers. IEEE Trans. Autom. Control 1981, 26, 926-936. [CrossRef]

10. Yao, Y.; Shahzad, N. Strong convergence of a proximal point algorithm with general errors. Optim. Lett. 2012, 6, 621-628. [CrossRef]

11. Yao, Y.-H.; Postolache, M.; Liou, Y.-C. Strong convergence of a self-adaptive method for the split feasibility problem. Fixed Point Theory Appl. 2013, 2013, 201. [CrossRef]

12. Dong, Q.-L.; Cho, Y.-J.; Rassias, T.-M. The projection and contraction methods for finding common solutions to variational inequality problems. Optim. Lett. 2018, 12, 1871-1896. [CrossRef]

13. Li, C.-L.; Jia, Z.-F. Expected residual minimization method for uncertain variational inequality problems. J. Nonlinear Sci. Appl. 2017, 10, 5958-5975. [CrossRef]

14. Chang, S.-S.; Zhu, Y.-G. On variational inequalities for fuzzy mappings. Fuzzy Sets Syst. 1989, 32, $359-367$. [CrossRef]

15. Huang, N.-J. Random generalized nonlinear variational inclusions for random fuzzy mappings. Fuzzy Sets Syst. 1999, 105, 437-444. [CrossRef]

16. Yao, Y.-H.; Liou, Y.C.; Yao, J.-C. Iterative algorithms for the split variational inequality and fixed point problems under nonlinear transformations. J. Nonlinear Sci. Appl. 2017, 10, 843-854. [CrossRef]

17. Lee, B.S.; Lee, G.M.; Cho, S.J.; Kim, D.S. A variational inequality for fuzzy mappings. In Proceedings of the Fifth Fuzzy Systems Association World Congress, Seoul, Korea, 4-9 July 1993; pp. 326-329.

18. Inuiguchi, $\mathrm{M}$; Ichihashi, $\mathrm{H}$; Kume, $\mathrm{Y}$. A solution algorithm for fuzzy linear programming with piecewise linear membership functions. Fuzzy Sets Syst. 1990, 34, 15-31. [CrossRef]

19. Hu, C.-F.; Fang, S.-C. Solving fuzzy inequalities with concave membership functions. Fuzzy Sets Syst. 1998, 99, 233-240. [CrossRef]

20. Fang, S.-C.; Hu, C.-F. Solving fuzzy variational inequalities. Fuzzy Optim. Decis. Mak. 2002, 1, $113-133$. [CrossRef]

21. Hu, C.-F. Solving Systems of Fuzzy Inequalities. Ph.D. Thesis, North Carolina State University, Raleigh, NC, USA, 1997.

22. $\mathrm{Hu}, \mathrm{C}$.-F. Solving variational inequalities in a fuzzy environment. J. Math. Anal. Appl. 2000, 249, 527-538. [CrossRef] 
23. Wang, H.-F.; Liao, H.-L. Variational inequality with fuzzy convex cone. J. Glob. Optim. 1999, 14, $395-414$. [CrossRef]

24. Wang, H.-F.; Liao, H.-L. Fuzzy resolution on the infeasibility of variational inequality. Eur. J. Oper. Res. 1998, 106, 198-203. [CrossRef]

25. Huang, N.-J. A new method for a class of nonlinear variational inequalities with fuzzy mappings. Appl. Math. Lett. 1997, 10, 129-133. [CrossRef]

26. Yao, Y.; Yao, J.-C.; Liou, Y.-C.; Postolache, M. Iterative algorithms for split common fixed points of demicontractive operators without priori knowledge of operator norms. Carpath. J. Math. 2018, 34, 459-466.

27. Yao, Y.; Liou, Y.-C.; Postolache, M. Self-adaptive algorithms for the split problem of the demicontractive operators. Optimization 2018, 67, 1309-1319. [CrossRef]

28. Yao, Y.-H.; Postolache, M.; Liou, Y.-C.; Yao, Z.-S. Construction algorithms for a class of monotone variational inequalities. Optim. Lett. 2016, 10, 1519-1528. [CrossRef]

29. Cubiotti, P.; Yao, J.-C. Discontinuous implicit quasi-variational inequalities with applications to fuzzy mappings. Math. Methods Oper. Res. 1997, 46, 213-228. [CrossRef]

30. Fang, S.-C.; Hu, C.-F.; Wang, H.-F.; Wu, S.-Y. Linear programming with fuzzy coefficients in constraints. Comput. Math. Appl. 1999, 37, 63-76. [CrossRef]

31. Jan, G.-M.; Sheu, R.-L.; Wu, S.-Y. Maximum feasibility problem for continuous linear inequalities with applications to fuzzy linear programming. Fuzzy Optim. Decis. Mak. 2003, 2, 297-316. [CrossRef]

32. Liou, Y.-C.; Wu, S.-Y.; Yao, J.-C. Bilevel decision with generalized semi-infinite optimization for fuzzy mappings as lower level problems. Fuzzy Optim. Decis. Mak. 2005, 4, 41-50. [CrossRef]

33. Yao, Y.; Qin, X.; Yao, J.-C. Projection methods for firmly type nonexpansive operators. J. Nonlinear Convex Anal. 2018, 19, 407-415.

34. Lan, H.-Y. An approach for solving fuzzy implicit variational inequalities with linear membership functions. Comput. Math. Appl. 2008, 55, 563-572. [CrossRef]

35. Wu, Z.Z.; Xu, J.P. A Class of Fuzzy Variational Inequality Based on Monotonicity of Fuzzy Mappings. Abstr. Appl. Anal. 2013, 2013, 854751. [CrossRef]

36. Tang, G.-J.; Zhao, T.; Wan, Z.P.; He, D.-X. Existence results of a perturbed variational inequality with a fuzzy mapping. Fuzzy Sets Syst. 2018, 331, 68-77. [CrossRef]

37. Fukushima, M. Equivalent differentiable optimization problems and descent methods for asymmetric variational inequality problems. Math. Program. 1992, 53, 99-110. [CrossRef]

38. Apostol, T.-M. Mathematical Analysis; Addison-Wesley: Boston, MA, USA, 1957.

39. Qi, L.; Shapiro, A.; Ling, C. Differentiability and semismoothness properties of integral functions and their applications. Math. Program. 2005, 102, 223-248. [CrossRef] 